\title{
Prevention of Venous Thromboembolism in Department of Veterans Affairs Hospitals
}

Jerome Herbers, MD, MBA Susan Zarter, BSN
Department of Veterans Affairs, Office of the Inspector General, Washington, DC.

The views and opinions expressed herein are those of the authors and do not necessarily state or reflect those of the Department of Veterans Affairs or the United States Government.

This article is a US Government work, and, as such, is in the public domain in the United States of America.

Disclosure: Nothing to report.

BACKGROUND: Pulmonary embolism (PE) is the most common preventable cause of death in hospitals, but many patients do not receive proven preventive therapies.

OBJECTIVE: To ascertain the extent to which inpatients received therapies for the prevention of venous thromboembolism (VTE).

DESIGN: Medical records review of a random sample of hospitalized patients, stratified by hospital teaching status.

SETTING: Department of Veterans Affairs (VA) acute-care hospitals.

PATIENTS: Two groups hospitalized $\geq 48$ hours during the year ending March 31, 2007: (1) all 4963 patients older than 74 years with a principal discharge diagnosis of heart failure; and (2) all 1448 patients with any discharge diagnosis of PE. MEASUREMENTS: Rate of VTE preventive care.

RESULTS: Sixty-three of the 100 randomly selected heart failure patients had adequate anticoagulation, 29 (46\%) of whom were taking warfarin for chronic conditions. For patients discharged with a PE diagnosis, records from all 330 nonteaching and 449 (40\%) teaching hospital cases were reviewed. Most cases (698; 90\%) were excluded because there was only a remote history of PE or the diagnosis was made prior to admission. Thirty-four of the 63 patients (54\%) with confirmed in-hospital PE and unequivocal VTE risk factors received appropriate preventive treatment. Thirty of the 66 patients (48\%) with missed opportunities for prevention had inappropriate mechanical prophylaxis or inadequate use of anticoagulation.

CONCLUSIONS: In hospitalized veterans with PE or at risk for VTE, missed opportunities for prevention were frequent and included inappropriate or inadequate interventions. Retrospective chart review was an inefficient method for identifying patients with in-hospital PE. Journal of Hospital Medicine 2010;5:E21-E25. (c) 2010 Society of Hospital Medicine.

\section{KEYWORDS: anticoagulants—therapeutic use, guideline adherence, hospitalization, pulmonary embolism-prevention and control,} venous thromboembolism—prevention and control.

Pulmonary embolism (PE) is the most common preventable cause of death in hospitals, ${ }^{1}$ accounting for approximately $10 \%$ of hospital deaths. Most cases of PE result from dislodged lower extremity thrombi, so that deep vein thrombosis (DVT) and PE are manifestations of the same disorder, venous thromboembolism (VTE). Even though the majority of hospitalized patients are at increased risk for VTE and proven preventive measures have long been available, most patients do not receive appropriate care. ${ }^{2}$

Recent surgery is a well-recognized risk factor for VTE, and surgeons have prescribed prophylactic therapies more consistently than other specialists. ${ }^{3}$ At the same time, prevention of VTE among hospitalized medical patients has been neglected. ${ }^{4}$ The American College of Chest Physicians recommends pharmacologic VTE prevention for most acutely ill medical patients, and advises prevention using mechanical devices when pharmacologic intervention is contraindicated. ${ }^{1}$
In Department of Veterans Affairs (VA) hospitals, compliance with preventive guidelines in surgical patients has been high. During October through December 2007, according to the Office of Quality and Performance, Veterans Health Administration, the national average for administration of VTE prophylaxis within 24 hours of surgery in VA hospitals was $92 \%$. No comparable systemwide performance measure has been applied for medical patients, but an assessment involving intensive care unit patients has been completed and plans are underway for an evaluation of anticoagulant use among all inpatients considered to be at increased risk.

We sought to determine the extent to which hospitalized VA medical patients receive VTE preventive care in accordance with evidence-based recommendations. Because quality of care may vary in hospitals based on teaching status, ${ }^{5}$ a secondary goal was to ascertain whether teaching and nonteaching facilities differ with respect to the delivery of care for VTE prevention.

2010 Society of Hospital Medicine DOI 10.1002/jhm.597

Published online in wiley InterScience (www.interscience.wiley.com). 


\section{Patient Populations Methods}

We examined compliance with accepted VTE clinical practice guidelines in 2 patient populations. First, the care of patients at risk for developing VTE was evaluated for evidence of appropriate preventive measures. Second, the care of patients who developed PE while hospitalized was evaluated for evidence of preventive therapy prior to the event.

We identified patients discharged from VA acute care hospitals during the period April 1, 2006 to March 31, 2007, excluding patients hospitalized for less than 48 hours. We also excluded hospitalizations of VA patients at military and private hospitals because oversight of the quality of care provided at those facilities is beyond the purview of the Inspector General.

We then defined 2 distinct populations:

1. Medical patients at increased risk for VTE. These patients were identified by: (1) age $\geq 75$ years at the time of admission; and (2) hospitalization with a principal discharge diagnosis of heart failure (International Classification of Diseases, 9th edition [ICD-9] code 428). Elderly heart failure patients were chosen because advanced age and heart failure are recognized VTE risk factors, medical inpatients have been identified as being neglected in hospital VTE prevention efforts, and the VA was conducting no systemwide assessment of this aspect of care.

2. Patients with established PE. These patients had any discharge diagnosis "pulmonary embolism and infarction" (ICD-9 codes 415.1 or 415.19 ), but those with the diagnostic code "personal history of venous thrombosis and embolism" (V12.51) were excluded.

Within each population, the discharge date defined an index hospitalization for evaluation. For patients discharged more than once with a qualifying diagnosis during the study period, we analyzed only the most recent hospitalization.

\section{Characterization of Facilities}

Hospitals were considered teaching hospitals if they were members of the Association of American Medical Colleges' Council of Teaching Hospitals and Health Systems (COTH) ${ }^{6}$ When COTH membership was through a Veterans Integrated Service Network, hospitals were judged to be teaching hospitals if they had 1 or more close university affiliations and/or management of medical inpatients by house staff.

\section{Patient Selection and Medical Record Review}

In order to ensure optimal representation of teaching and nonteaching hospitals, we stratified patients with increased VTE risk and those with diagnosed PE according to hospital teaching status, thereby creating 4 groups:

1. Patients at increased risk for VTE-teaching hospitals;

2. Patients at increased risk for VTE-nonteaching hospitals;

3. PE patients-teaching hospitals;

4. PE patients-nonteaching hospitals.
Within each group we assigned a random number to each patient, ordered the patients by random number, and selected patients sequentially until 50 patients were identified or no further eligible patients were available. For the heart failure patients, we assumed that all were at risk for VTE and required prophylaxis. For the group of patients with established PE, we excluded patients if the diagnosis was made prior to admission or in the first 2 hospital days, if there were no acute signs and symptoms and the diagnosis was chronic PE, or if there were no imaging studies or postmortem findings in support of the diagnosis.

In both groups, we assessed patients' records for VTE risk factors, evidence of preventive care, and contraindications. ${ }^{7}$ We considered pertinent VTE risk factors to be those included with published guidelines. ${ }^{1}$ In the care of patients with established $\mathrm{PE}$, designation of adequate prophylactic therapy required at least 24 hours of treatment prior to diagnosis.

Appropriate VTE prophylaxis was defined as anticoagulant medications or, in the case of contraindications to anticoagulation, mechanical compression devices applied to the lower extremities with or without antiembolism stockings. Any administration of warfarin, low-molecular-weight heparin, or heparin by infusion, was considered adequate. Prophylaxis with subcutaneous unfractionated heparin was considered adequate only if at least 5000 units was administered 3 times daily. ${ }^{8}$ Aspirin and other antiplatelet agents were not considered to be anticoagulants.

We characterized hospitalizations of at-risk individuals as missed opportunities for prevention if there were no contraindications to treatment and no evidence that adequate prophylactic therapy was provided.

\section{Data Analysis}

To determine sample size for each of the 2 study populations, we assumed the baseline rate of compliance with recommendations for VTE prophylaxis among medical inpatients to be 0.4 to $0.5 .{ }^{4}$ We further assumed that an observed compliance rate of 0.7 would be indicative of an important difference compared with published results (rate difference, 0.2-0.3). With a Type 1 (alpha) error of 0.025 (1-tailed), approximately 90 patients are required for a 0.9 probability of detecting a difference of at least $0.25 .^{9}$

Comparisons between teaching and nonteaching hospitals were analyzed using chi-square tests. Confidence intervals for estimates of overall compliance were calculated using a normal approximation to the binomial distribution. ${ }^{10}$

\section{Results}

\section{Medical Patients at Increased Risk for VTE}

We identified 4963 patients age 75 and older discharged after at least 2 days of acute hospitalization for heart failure: 3437 from 73 teaching hospitals and 1526 from 58 nonteaching hospitals. The 100 patients randomly selected for 
TABLE 1. Treatment of Inpatients at Increased Risk for Venous Thromboembolism at Acute Care VA Hospitals, April 1, 2006 through March 31, 2007

\begin{tabular}{lrrr} 
& \multicolumn{3}{c}{ Hospital Type } \\
\cline { 2 - 4 } & Total & Teaching & Nonteaching \\
\hline Number of patients & 4963 & 3437 & 1526 \\
Randomly selected patients at risk & 100 & 54 & 46 \\
Received prophylactic anticoagulation & 63 & 34 & 29 \\
Missed opportunities for prevention & 37 & 20 & 17 \\
Percent (95\% confidence interval) & $37(27-47)$ & $37(23-51)$ & 37 (22-52) \\
\hline Abbreviation: VA, Department of Veterans Affairs. & & & \\
\hline
\end{tabular}

review ranged in age from 75 to 94 (median, 82) and had hospitalizations of 3 to 41 days (median, 6). Ninety-eight were male. In this group of patients, we found 63 with evidence of adequate pharmacologic VTE prevention and 37 for which opportunities for prevention were not realized. At teaching hospitals, anticoagulation was effected with warfarin (13 cases), heparin (11), and enoxaparin (10). At nonteaching hospitals anticoagulants included warfarin (16), enoxaparin (11), and heparin (2). Twenty-nine of the 63 patients who received anticoagulation (46\%) were admitted while taking warfarin for chronic conditions. Teaching and nonteaching hospitals did not differ with respect to missed opportunities for prevention of VTE $(37 \%$ in each group; Table 1).

\section{Patients With Established PE}

We identified 1448 acute hospitalizations of at least 2 days duration for patients with PE, 1118 from 72 teaching hospitals and 330 from 51 nonteaching hospitals. We reviewed 779 medical records, 449 (40.2\%) teaching cases and all 330 nonteaching cases. Chart review was completed after all nonteaching cases had been reviewed. In only $8.2 \%$ (64) of reviewed cases was the diagnosis of acute PE made after the first 2 hospital days and with accompanying objective evidence of VTE. Most cases (698; 89.6\%) were excluded because there was only a remote history of PE or the diagnosis was made prior to admission. Additional cases (17; $2.2 \%$ ) were excluded because the diagnosis was made during the first 2 hospital days; there were no acute signs and symptoms and the diagnosis was chronic PE; or there was no confirmation by computed tomography or ventilationperfusion scans, lower extremity ultrasonography in the setting of consistent clinical findings, or autopsy.

The 64 patients with confirmed in-hospital PE ranged in age from 44 to 85 years (median, 65) and had hospitalizations of 4 to 53 days (median, 16). Sixty-three were male. One of these patients had no definite risk factors for VTE and was ambulatory when acute symptoms occurred. Among the 63 patients who had unequivocal VTE risk factors, $34(54 \%)$ received appropriate prophylactic treatment,

\begin{tabular}{lccc} 
TABLE 2. Patients With a Discharge Diagnosis of \\
PE at Acute Care VA Hospitals, April 1, $\mathbf{2 0 0 6}$ \\
Through March 31, 2007 & \multicolumn{3}{c}{ Hospital Type } \\
\cline { 2 - 4 } & Total & Teaching & Nonteaching \\
\cline { 2 - 4 } & 1448 & 1118 & 330 \\
\hline $\begin{array}{l}\text { Number of patients } \\
\text { Randomly selected patients }\end{array}$ & 779 & 449 & 330 \\
$\begin{array}{l}\text { Documented in-hospital } \\
\text { pulmonary embolism }\end{array}$ & 64 & 47 & 17 \\
$\begin{array}{l}\text { No definite VTE risk factors } \\
\text { Received prophylactic anticoagulation }\end{array}$ & 30 & 0 & 1 \\
Anticoagulation contraindicated, & 4 & 20 & 10 \\
$\quad$ received mechanical prophylaxis & & 4 & 0 \\
$\begin{array}{l}\text { Missed opportunities for prevention } \\
\text { Percent (95\% confidence interval) }\end{array}$ & $45(32-58)$ & $49(30-68)$ & 35 (12-58) \\
\hline
\end{tabular}

Abbreviations: PE, pulmonary embolism; VA, Department of Veterans Affairs; VTE, venous thromboembolism.

and 29 (46\%) received inadequate or no preventive therapy (Table 2). There was no significant difference between teaching and nonteaching hospitals with respect to missed opportunities for prevention ( $49 \%$ vs. $35 \% ; P \geq 0.3$ ).

Anticoagulants used at teaching hospitals included heparin (15 cases), enoxaparin (4), and warfarin (1), while at nonteaching hospitals enoxaparin (7) and heparin (3) were used.

Each of the 10 patients who received no anticoagulation had the VTE risk factor of recent immobility prior to PE. Nine of the 10 had active malignancies, and 4 of these had undergone recent surgery. None had evidence of hypercoagulable states (factor V Leiden, lupus anticoagulant, or anticardiolipin antibodies). Five of the 10 patients died in the year following pulmonary embolism, 3 prior to discharge or within 2 weeks of discharge.

\section{Missed Opportunities for Prevention}

Among the 66 patients whose hospitalizations were characterized as missed opportunities for prevention, 30 received no pharmacologic VTE prevention despite having no contraindications. Seven patients had contraindications to pharmacologic prophylaxis, but none of these patients had contraindications to mechanical prophylaxis. An additional 18 patients received mechanical prophylaxis only despite having no contraindications to anticoagulation. Eleven patients received inadequate heparin regimens with or without mechanical prophylaxis (Table 3).

\section{Discussion}

Based on a random sample of 4963 elderly heart failure patients admitted to VA hospitals during a 1-year period, we estimated that $63 \%$ received recommended interventions aimed at preventing VTE. Although differences in methodology limit comparisons with published reports, this rate is similar to those observed at individual hospitals, ${ }^{11-13}$ in 


\begin{tabular}{|c|c|c|c|}
\hline & \multicolumn{3}{|c|}{ Hospital Type } \\
\hline & Total & Teaching & Nonteaching \\
\hline Number of patients & 66 & 43 & 23 \\
\hline No contraindications, no prophylaxis & 30 & 18 & 12 \\
\hline $\begin{array}{l}\text { Anticoagulation contraindicated, } \\
\text { no mechanical prophylaxis }\end{array}$ & 7 & 3 & 4 \\
\hline $\begin{array}{l}\text { No contraindication to anticoagulation, } \\
\text { mechanical prophylaxis only }\end{array}$ & 18 & 13 & 5 \\
\hline $\begin{array}{l}\text { Inadequate heparin regimen, } \\
\text { no mechanical prophylaxis }\end{array}$ & 9 & 7 & 2 \\
\hline $\begin{array}{l}\text { Inadequate heparin regimen, } \\
\text { mechanical prophylaxis }\end{array}$ & 2 & 2 & 0 \\
\hline
\end{tabular}

large multicenter registries of patients with DVT or at risk for VTE, ${ }^{14,15}$ and in a recent multinational cross-sectional study. $^{16}$ Notably, chronic outpatient anticoagulation that was continued during hospitalization accounted for nearly one-half of patients receiving preventive care. Compliance did not differ between teaching and nonteaching hospitals.

In a complementary approach to examining the extent of preventive care, we identified 1448 patients discharged with a diagnosis of PE. Most of these patients were excluded because they did not have a new event while hospitalized. Eleven (17\%) of the 64 patients with confirmed in-hospital PE received no preventive care before the event. An additional 18 (28\%) received suboptimal heparin regimens or mechanical prophylaxis in the absence of contraindications to anticoagulation. As with the patients at risk for VTE, patients with established PE at teaching and nonteaching hospitals received similar rates of preventive care. Contrary to our expectation, the observed difference in rates between types of hospitals favored nonteaching hospitals. However, the sample size for this comparison was small and the difference did not reach statistical significance.

This study's population-based approach permits conclusions about the performance of the VA's entire system of acute care hospitals. The results indicate that proven preventive therapies are often neglected at VA hospitals, but overall performance is probably comparable to other settings. VA employs an extensively implemented electronic medical record (EMR) and superior performance might have been expected. However, these results suggest limitations in the EMR as it is currently deployed. Successful efforts probably require a multifaceted approach incorporating decision support and institutional standardization. ${ }^{17}$

Several additional findings warrant comment. Patients with malignancies accounted for 9 of 10 patients who had PE after receiving no prior anticoagulation. Recent surgery was also a contributing factor for 4 of these cancer patients.
Although both cancer and surgery are well-known risk VTE factors, clinicians may not appreciate the extremely high risk associated with the combination. ${ }^{18}$ Particular effort may be warranted to ensure prophylaxis in this group, and more intensive measures may be necessary.

These results reveal several barriers to the accurate retrospective measurement of preventable inpatient PE. First, the use of discharge diagnoses to monitor the occurrence of inpatient PE is fraught with hazard. In this study, even after excluding patients with a discharge diagnostic code indicating a past history of PE, very few identified patients in fact had an acute or recent event. In addition, many patients were clearly admitted after having the onset of symptoms as outpatients. Further, reliance on discharge diagnoses alone can lead to the inclusion of patients with a presumptive diagnosis made without the advantage of imaging studies or postmortem examination. Although we overcame these barriers through careful record review and strict diagnostic criteria, our results suggest that efficient performance improvement efforts may require ongoing concurrent review.

There are several limitations of this study. First, we excluded PE patients whose diagnoses were made before the third hospital day. Some of these patients may have had events attributable to recent prior hospitalizations and should have received VTE prophylaxis. Second, we considered preventive measures applied at least 24 hours prior to $\mathrm{PE}$ to be acceptable evidence of prevention, potentially neglecting prior periods without treatment that might confer increased risk. Bias due to either of these limitations could exaggerate the compliance rates we report. Finally, the retrospective design of this study did not allow for consistent assessments of whether patients had the risk factor of immobility. Nevertheless, immobility was obvious for the 10 patients with PE who had no prior anticoagulation, all of whom had 2 or more risk factors.

Despite an acknowledged need for improvements in clinical practice, past efforts have had mixed results. For instance, in 1 study at a hospital with a well-established EMR, computer alerts led to substantial improvement in the use of preventive measures and in VTE outcomes, but overall compliance remained low. ${ }^{19}$ On the other hand, a multidisciplinary approach can achieve marked reductions in preventable VTE events. ${ }^{20}$ Key elements of such an approach are a simplified risk assessment tool and concurrent monitoring of patient treatments and outcomes. The Agency for Healthcare Research and Quality has recently published a guide that outlines strategies for achieving "breakthrough levels of improvement" in the prevention of VTE. $^{21}$

In conclusion, this population-based study of hospitalized veterans with PE or at risk for VTE found compliance comparable to rates in published reports. Missed opportunities for prevention included inappropriate and inadequate interventions. Using discharge diagnoses to monitor the occurrence of inpatient PE is of limited value, and efficient 
performance improvement efforts may require ongoing concurrent review.

\section{Acknowledgements}

The authors thank Greg Maynard, MD, MS for advice on study design, and Deborah Howard, RN, for assistance with medical record review.

\section{Address for correspondence and reprint requests:}

Jerome Herbers, MD, MBA, Department of Veterans Affairs, 54AA, Washington, DC 20420; Telephone: 202-461-4658; Fax: 202565-8476; E-mail: jerome.herbers@va.gov Received 30 September 2008; revision received 2 July 2009; accepted 25 July 2009.

\section{References}

1. Geerts WH, Pineo GF, Heit JA. Prevention of venous thromboembolism. The Seventh ACCP Conference on Antithrombotic and Thrombolytic Therapy. Chest. 2004;126:338S-400S.

2. Amin A, Stemkowski S, Lin J, Yang G. Thromboprophylaxis rates in US medical centers: success or failure? J Thromb Haemost. 2007;5:1610-1616.

3. Michota FA. Preventing venous thromboembolism in surgical patients. Cleve Clin J Med. 2006;73:S88-S94.

4. Goldhaber SZ, Turpie AGG. Prevention of venous thromboembolism among hospitalized medical patients. Circulation. 2005;111:e1-e3.

5. Landon BE, Normand SL, Lessler A. Quality of care for the treatment of acute medical conditions in US hospitals. Arch Intern Med. 2006;166:2511-2517.

6. Association of American Medical Colleges (AAMC). Council of Teaching Hospitals and Health Systems (COTH). Available at: http://www.aamc. org/members/coth. Accessed August 2009.

7. Contraindications are those described in the Institute for Clinical Systems Improvement Health Care Guideline: Venous Thromboembolism Prophylaxis. 4th ed. June 2007, pp 13-14. Available at: http://www. icsi.org/guidelines_and_more/gl_os_prot/cardiovascular/venous_thromboem bolism_prophylaxis/venous_thromboembolism_prophylaxis_5.html. Accessed August 2009

8. Nicolaides AN, Fareed J, Kakkar AK, et al. Prevention and treatment of venous thromboembolism. International Consensus Statement. Int Angiol. 2006;25:101-161.

9. Hulley SB, Cummings SR. Designing Clinical Research. Baltimore, MD: Williams \& Wilkins; 1988.
10. Fleiss JL, Levin B, Paik MC. Statistical Methods for Rates and Proportions. 3rd ed. Hoboken, NJ: Wiley-Interscience; 2003;28, 54.

11. Goldhaber SZ, Dunn K, MacDougall RC. New onset of venous thromboembolism among hospitalized patients at Brigham and Women's Hospital is caused more often by prophylaxis failure than by withholding treatment. Chest. 2000;118;1680-1684.

12. Peterman CM, Kolansky DM, Spinler SA. Prophylaxis against venous thromboembolism in acutely ill medical patients: an observational study. Pharmacotherapy. 2006;26:1086-1090.

13. Dorfman M, Chan SB, Maslowski C. Hospital-acquired venous thromboembolism and prophylaxis in an integrated hospital delivery system. J Clin Pharm Ther. 2006;31:455-459.

14. Goldhaber SZ, Tapson VF; DVT FREE Steering Committee. A prospective study of 5,451 patients with ultrasound-confirmed deep vein thrombosis. Am J Cardiol. 2004;15:259-262.

15. Tapson VF, Decousus H, Pini M, et al. Venous thromboembolism prophylaxis in acutely ill hospitalized medical patients: findings from the International Medical Prevention Registry on Venous Thromboembolism. Chest. 2007;132:936-945.

16. Cohen AT, Tapson VF, Bergmann JF, et al. Venous thromboembolism risk and prophylaxis in the acute hospital care setting. Lancet. 2008;371: 387-394.

17. Maynard GA. Medical admission order sets to improve deep vein thrombosis prevention: a model for others or a prescription for mediocrity? J Hosp Med. 2009;4:77-80.

18. Spyropoulos AC, Brotman DJ, Amin AN, Deitelzweig SB, Jaffer AK, McKean SC. Prevention of venous thromboembolism in the cancer surgery patient. Cleve Clin J Med. 2008;75(suppl 3):S17-S26.

19. Baroletti S, Munz K, Sonis J, et al. Electronic alerts for hospitalized highVTE risk patients not receiving prophylaxis: a cohort study. J Thromb Thrombolysis. 2008;25:146-150.

20. Maynard G, Jenkins I, Stone S, et al. Prevention of hospital-acquired venous thromboembolism: prospective validation of a VTE risk assessment model and protocol. Society of Hospital Medicine 2008 National Meeting. Electronic citation abstract \#52, page 29. Available at: http://www.hospital medicine.org/Paperless2008/PDFs/Additional_Info/SHM08_Abstracts.pdf. Accessed August 2009

21. Maynard G, Stein J. Preventing Hospital-Acquired Venous Thromboembolism: A Guide for Effective Quality Improvement. AHRQ Publication No. 08-0075. Rockville, MD: Agency for Healthcare Research and Quality; 2008. 